\title{
Status do conhecimento tradicional sobre camarões peneídeos e carcinofauna acompanhante no Brasil: uma revisão bibliográfica
}

Este estudo tem como objetivo analisar o status de informações presentes na literatura relacionada ao conhecimento de pescadores sobre camarões peneídeos e carcinofauna acompanhante de áreas costeiras e na plataforma continental do Brasil. Para obtenção dos dados foi realizado um levantamento bibliográfico de artigos, teses e dissertações sobre o tema. No total foram encontrados 12 estudos, sendo oito (08) artigos publicados em revistas com corpo editorial, três (03) dissertações de mestrado e uma (01) tese de doutorado, distribuídos pelas regiões Sudeste $(n=06)$; Nordeste $(n=06)$ e Sul $(n=02)$ do país. O número de estudos por regiões $(n=14)$ é maior que o número total de trabalhos $(n=12)$, pois uma mesma pesquisa era realizada em mais de uma região. No total foram registradas sete famílias distribuídas em 11 gêneros e 13 espécies de crustáceos. Os camarões sete-barbas (Xiphopenaeus kroyeri), branco (Litopenaeus schimitti) e rosa (Farfantepenaeus brasiliensis) foram os mais abordados nos estudos. Outros crustáceos como, caranguejos, siris, lagostas, ermitão (búzios/caramujos) e camarão carídeo (mãezinha/camarão-espinho) foram citados como fauna acompanhante da pesca de camarões. A partir desta revisão é possível identificar que os crustáceos mais descritos via conhecimento tradicional são também os recursos pesqueiros mais explorados comercialmente. Desta forma, a compreensão do saber local de comunidades pesqueiras pode ser uma ferramenta útil na obtenção de informações de ocorrência de espécies de crustáceos para estatística pesqueira o que contribui com ações de manejo da pesca de áreas costeiras e da plataforma continental brasileira.

Palavras-chave: Pesca artesanal; Comunidade pesqueira; Crustacea; Etnobiologia; Etnoecologia.

\section{Status of traditional knowledge about penaeidae shrimps and accompanying carcinofauna in Brazil: a bibliographic review}

\begin{abstract}
This study aims to analyze the status of information present in the literature related to the knowledge of fishermen about peneid shrimp and carcinofauna accompanying in coastal areas and on the continental shelf of Brazil. To obtain the data, a bibliographic survey of articles, theses and dissertations on the topic was carried out. In total, 12 studies were found, of which eight (08) articles were published in journals with an editorial board, three (03) master's dissertations and one (01) doctoral thesis, distributed throughout the Southeast $(n=06)$; Northeast $(n=06)$ and South $(n=02)$ of the country. The number of studies by region $(n=14)$ is greater than the total number of studies $(n=12)$, since the same research was carried out in more than one region. In total, seven families were registered, distributed in 11 genera and 13 species of crustaceans. The Atlantic seabob shrimp (Xiphopenaeus kroyeri), white shrimp (Litopenaeus schimitti) and pink shrimp (Farfantepenaeus brasiliensis) were the most addressed in the studies. Other crustaceans, such as crabs, lobsters, hermit (shells/snails) and spineshrimp (mother/thorn shrimp) were mentioned as fauna accompanying shrimp fishing. From this review it is possible to identify that the crustaceans most described via traditional knowledge are also the most commercially exploited fishing resources. Thus, understanding the local knowledge of fishing communities can be a useful tool in obtaining information on the occurrence of crustacean species for fisheries statistics, which contributes to fisheries management actions in coastal areas and on the Brazilian continental shelf.
\end{abstract}

Keywords: Artisanal fishery; Fishery community; Crustacea; Ethnobiology; Ethnoecology.

Topic: Uso de Recursos Naturais

Reviewed anonymously in the process of blind peer.
Received: 03/01/2021

Approved: 28/01/2021
Adriane Cristina Araújo Braga (iD

Universidade Federal do Espírito Santo, Brasil

http://lattes.cnpq.br/4795754965416046

http://orcid.org/0000-0002-2790-8794

dricrab@yahoo.com.br

Camilah Antunes Zappes (D)

Universidade Federal Fluminense, Brasil

http://lattes.cnpq.br/0217232489124641

http://orcid.org/0000-0002-5486-6577

camilahaz@yahoo.com.br
Referencing this:

BRAGA, A. C. A.; ZAPPES, C. A.. Status do conhecimento tradicional sobre camarões peneídeos e carcinofauna acompanhante no Brasil: uma revisão bibliográfica. Revista Ibero Americana de Ciências Ambientais, v.12, n.1, p.641-650, 2021. DOI:

http://doi.org/10.6008/CBPC2179-6858.2021.001.0051 


\section{INTRODUÇÃO}

Ao longo da costa brasileira a pesca artesanal é praticada diariamente por homens e mulheres de comunidades pesqueiras cujo principal propósito é a subsistência e obtenção da renda familiar (SILVANO, 2004). Esta prática diária da atividade e a vivência dos atores locais junto ao ambiente marinho proporcionam um acúmulo de conhecimento sobre a biologia e ecologia de espécies marinhas e sua relação com as variáveis ambientais (MOURÃO et al., 2006; OLIVEIRA et al., 2016; ABREU et al., 2020). Este saber é denominado por conhecimento tradicional sendo elaborado por meio de observações contínuas de pescadores e pescadoras sobre a natureza, e compartilhado entre gerações (PAZ et al., 1996; HANAZAKI et al., 2007; FRIESINGER et al., 2010; ZAPPES et al., 2016).

Dentre as abordagens que envolvem o conhecimento tradicional de populações humanas sobre processos biológicos, ecológicos e culturais, a etnobiologia e etnoecologia são áreas da ciência que possibilitam a compreensão das interações do ser humano com o ambiente (DIEGUES, 2004). Estas duas áreas de conhecimento permitem a junção de informações obtidas por pesquisadores e comunidades o que pode contribuir em ações voltadas ao manejo da pesca e conservação de recursos pesqueiros (MUSIELLOFERNANDES et al., 2017; 2018; CÔRTES et al., 2019).

Dentre os principais recursos pesqueiros, os crustáceos são considerados importantes uma vez que apresentam elevado valor econômico, podem contribuir para a subsistência de uma população; além de gerar emprego e renda para comunidades pesqueiras (IBAMA, 1994; BOTELHO et al., 2000; JANKOWSKY et al., 2006; MAGALHÃES et al., 2012; CÔRTES et al., 2018). No Brasil, o camarão como importante recurso pesqueiro, é capturado pela pesca de arrasto (DI BENEDITTO, 2001; SOUZA et al., 2009). Esta pescaria apresenta baixa seletividade em que há captura de elevada biomassa de indivíduos de outras espécies definida como fauna acompanhante composta por peixes e macroinvertebrados (GRAÇA-LOPES et al., 2002; BRANCO et al., 2004; BRANCO et al., 2006; DIAS NETO, 2011; SEDREZ et al., 2013). Dessa fauna acompanhante, a maior parte é considerada de baixo valor comercial sendo devolvida ao mar debilitada ou morta (GRAÇA-LOPES et al., 2002; BRANCO et al., 2004; EUTRÓPIO, 2009).

O conhecimento tradicional relacionado aos aspectos biológicos e ecológicos de espécies-alvo da pesca é importante para o direcionamento de estudos científicos que visam contribuir em políticas públicas voltadas ao manejo pesqueiro (HANAZAKI, 2002; BATISTA et al., 2004; MUSIELLO-FERNANDES et al., 2018; CÔRTES et al., 2019). Além disso, o amplo conhecimento adquirido por pescadores e pescadoras artesanais sobre a biologia e ecologia das espécies de crustáceos pode auxiliar no levantamento da diversidade desse táxon, no entendimento sobre a perda da biodiversidade e a importância da conservação junto às comunidades pesqueiras (BRAGA et al., 2018).

No Brasil, ainda são incipientes os estudos voltados à etnobiologia e etnoecologia de camarões peneídeos e sua carcinofauna o que demonstra a importância em se identificar as lacunas de conhecimento envolvendo está temática (BRAGA et al., 2018). Desta forma, este artigo tem como objetivo analisar o status de informações presentes na literatura relacionada ao conhecimento tradicional de pescadores e pescadoras 
artesanais sobre esses camarões e a carcinofauna acompanhante da sua pescaria realizada em áreas costeiras e na plataforma continental do Brasil.

\section{MATERIAIS E MÉTODOS}

A fim de obter os dados foi realizado levantamento bibliográfico especificamente de estudos relacionados à etnobiologia e etnoecologia de camarões peneídeos e sua carcinofauna ao longo da plataforma continental brasileira. A listagem bibliográfica foi realizada a partir das bases de dados 'Biblioteca Digital Brasileira de Teses e Dissertações (BDTD)', 'ISI Web of Science', 'Scirus', 'Google Scholar', 'Scielo', 'Science Direct', 'Scopus', e pelas plataformas 'Lattes' (vinculada ao Conselho Nacional de Desenvolvimento Científico e Tecnológico - CNPq) e 'Researchgate'. Ainda, uma busca foi realizada em sites de universidades pública e privada do Brasil.

Para a realização do levantamento bibliográfico foi utilizada palavras-chave em inglês, português e espanhol, sendo: 'Etnoecologia + crustáceos + camarão + peneídeos + carcinofauna', 'Etnobiologia + crustáceos + camarão + peneídeos + carcinofauna', 'Ecologia Humana + crustáceos', 'Conhecimento Ecológico Local + crustáceos', 'Conhecimento tradicional + crustáceos', 'Saber local + crustáceos', 'Interações + crustáceos', 'Pesca artesanal + crustáceos', 'Pescadores artesanais + crustáceos', 'Entrevistas + crustáceos', 'Relatos + crustáceos'; ‘'Brasil + crustáceos + conhecimento tradicional’. Após a pesquisa foram considerados apenas os estudos em que o objetivo principal envolvia o uso do conhecimento tradicional de pescadores e pescadoras sobre a biologia e ecologia das espécies de camarões peneídeos e sua carcinofauna no litoral brasileiro.

\section{RESULTADOS}

Em todos os estudos encontrados os métodos utilizados para obtenção dos dados de conhecimento tradicional envolvendo a temática ocorreu principalmente por meio de realização de entrevistas podendo ser utilizado também diário de campo, observação participante, questionário e/ou gravações de relatos a fim de compreender a percepção local.

De acordo com o levantamento bibliográfico foram contabilizados 12 trabalhos, sendo oito (08) artigos publicados em revistas com corpo editorial, três (03) dissertações de mestrado e uma (01) tese de doutorado. Destes estudos, a maioria foi realizada nas regiões Sudeste $(n=06)$ e Nordeste $(n=06)$, seguida do Sul ( $n=02$ ), e nenhum registro na região Norte (Figura 1). Dois artigos (MUSIELLO-FERNANDES et al., 2017; 2018) abrangeram duas regiões (Nordeste e Sudeste) (Tabela 1).

Nos estudos, os entrevistados mencionaram os camarões peneídeos como espécies-alvo da pesca, sendo descritos: o camarão sete-barbas $(n=12)$; o camarão branco $(n=06)$ e o camarão rosa $(n=04)$ (Figura 2). Outros crustáceos como, caranguejos, siris, lagostas, ermitão (búzio/caramujo) e camarão carídeo (mãezinha/camarão-espinho) foram citados como carcinofauna acompanhante (Figura 2, Tabela I). Nesta revisão foram registradas nove famílias (Aethridae, Diogenidae, Leucosiidae, Lysmatidae, Palaemonidae, Palinuridae, Penaeidae, Portunidae, Scyllaridae) distribuídas em 13 espécies de crustáceos citados pelos 
pescadores artesanais e uma (1) não identificada (Tabela 1).

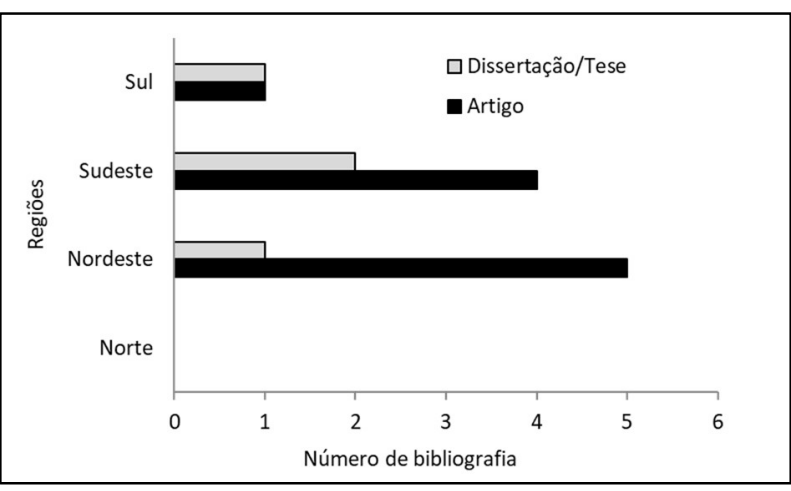

Figura 1: Distribuição de estudos referentes à etnobiologia e etnoecologia de camarões peneídeos e carcinofauna por região brasileira.

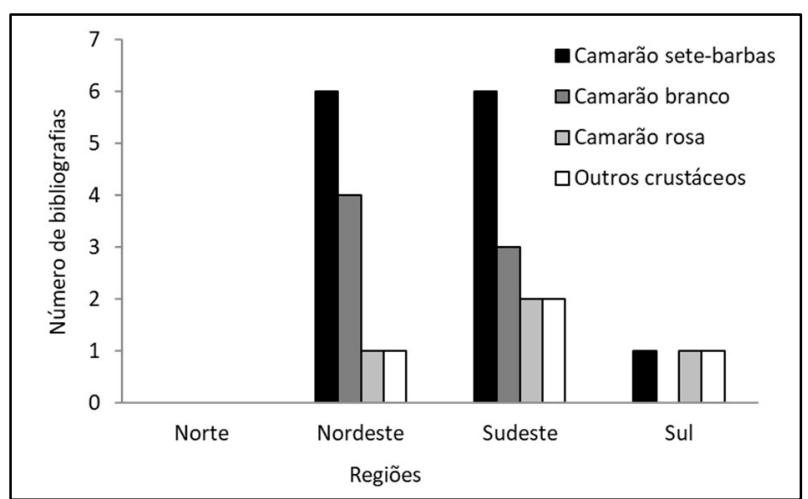

Figura 2: Camarões peneídeos de interesse econômico e carcinofauna citados nos estudos por região brasileira.

Tabela 1: Etnoespécies de camarões peneídeos e carcinofauna citadas por pescadores artesanais nos estudos.

\begin{tabular}{|c|c|c|c|c|}
\hline Família & Nome científico & Etnoespécies & Região/ Área de estudo & Literatura \\
\hline $\begin{array}{l}\text { Penaeidae } \\
\text { Rafinesque, } 1815\end{array}$ & $\begin{array}{ll}\text { Xiphopenaeus } & \text { kroyeri } \\
\text { (Heller, 1862) } & \\
\end{array}$ & $\begin{array}{l}\text { Camarão sete- } \\
\text { barbas }\end{array}$ & $\begin{array}{l}\text { SUL } \\
\text { Penha, Santa Catarina }\end{array}$ & BAIL et al., 2007. \\
\hline $\begin{array}{l}\text { Penaeidae } \\
\text { Rafinesque, } 1815 \\
\\
\text { Palinuridae } \\
\text { Latreille, } 1802 \\
\text { Scyllaridae } \\
\text { Latreille, } 1825\end{array}$ & $\begin{array}{l}\text { Xiphopenaeus kroyeri } \\
\text { (Heller, 1862) } \\
\text { Litopenaeus schimitti } \\
\text { (Burkenroad, 1936) } \\
\text { Farfantepenaeus } \\
\text { brasiliensis (Latreille, } \\
\text { 1817) } \\
\text { Farfantepenaeus } \\
\text { subtilis (Pérez-Farfante, } \\
\text { 1967) } \\
\text { Panulirus sp. White, } \\
\text { 1847 } \\
\text { Scyllarus aequinoctialis } \\
\text { (Lund, 1793) }\end{array}$ & $\begin{array}{l}\text { Camarão sete- } \\
\text { barbas } \\
\text { Camarão branco } \\
\text { Camarão rosa } \\
\text { Camarão rosa } \\
\text { Lagosta espinho } \\
\text { Lagosta sapata }\end{array}$ & $\begin{array}{l}\text { NORDESTE: } \\
\text { Itacaré, BA }\end{array}$ & $\begin{array}{l}\text { ALARCON et al., } \\
2009 .\end{array}$ \\
\hline $\begin{array}{l}\text { Penaeidae } \\
\text { Rafinesque, } 1815\end{array}$ & $\begin{array}{l}\text { Farfantepenaeus } \\
\text { paulensis (Pérez- } \\
\text { Farfante, 1967) } \\
\text { Callinectes sapidus } \\
\text { Rathbun, } 1896\end{array}$ & $\begin{array}{l}\text { Camarão rosa } \\
\text { Siri }\end{array}$ & $\begin{array}{l}\text { SUL: } \\
\text { litoral Norte do Rio Grande do Sul: } \\
\text { Balneário Pinhal, Cidreira, } \\
\text { Tramandaí, Imbé, Xangri-lá, Capão da } \\
\text { Canoa, Osório, Maquiné, Terra de } \\
\text { Areia, Arroio do Sal, Torres }\end{array}$ & PERUCCHI, 2013. \\
\hline $\begin{array}{l}\text { Penaeidae } \\
\text { Rafinesque, } 1815\end{array}$ & $\begin{array}{l}\text { Xiphopenaeus kroyeri } \\
\text { (Heller, 1862) }\end{array}$ & $\begin{array}{l}\text { Camarão sete- } \\
\text { barbas }\end{array}$ & $\begin{array}{l}\text { SUDESTE: } \\
\text { Praia Grande, Mongaguá, Itanhaém e } \\
\text { Peruíbe -SP }\end{array}$ & GOMES, 2015. \\
\hline $\begin{array}{l}\text { Penaeidae } \\
\text { Rafinesque, } 1815\end{array}$ & $\begin{array}{l}\text { Xiphopenaeus kroyeri } \\
\text { (Heller, 1862) } \\
\text { Litopenaeus schimitti } \\
\text { (Burkenroad, 1936) }\end{array}$ & $\begin{array}{l}\text { Camarão sete- } \\
\text { barbas } \\
\text { Camarão branco }\end{array}$ & $\begin{array}{l}\text { SUDESTE: } \\
\text { Barra do Una, Peruíbe (SP/ Brasil) } \\
\text { (litoral sul) }\end{array}$ & $\begin{array}{l}\text { ZEINEDDINE et } \\
\text { al., } 2015 .\end{array}$ \\
\hline $\begin{array}{l}\text { Penaeidae } \\
\text { Rafinesque, } 1815\end{array}$ & $\begin{array}{l}\text { Xiphopenaeus kroyeri } \\
\text { (Heller, 1862) }\end{array}$ & $\begin{array}{l}\text { Camarão } \\
\text { barbas }\end{array}$ & $\begin{array}{l}\text { SUDESTE: } \\
\text { Anchieta e Barra Nova (ES) e } \\
\text { NORDESTE: } \\
\text { Barras de Caravelas e Santa Cruz de } \\
\text { Cabralia (BA) }\end{array}$ & $\begin{array}{l}\text { MUSIELLO- } \\
\text { FERNANDES et al., } \\
2017 .\end{array}$ \\
\hline $\begin{array}{l}\text { Lysmatidae } \\
\text { Dana, } 1852 \\
\text { Diogenidae } \\
\text { Ortmann, } 1892 \\
\text { Portunidae } \\
\text { Rafinesque, } 1815 \\
\end{array}$ & $\begin{array}{l}\text { Xiphopenaeus kroyeri } \\
\text { (Heller, 1862) } \\
\text { Litopenaeus schimitti } \\
\text { (Burkenroad, 1936) } \\
\text { Farfantepenaeus } \\
\text { brasiliensis (Latreille, } \\
\text { 1817) } \\
\text { Exhippolysmata } \\
\text { oplophoroides } \\
\text { (Holthuis, 1948) } \\
\end{array}$ & $\begin{array}{l}\text { Camarão sete- } \\
\text { barbas } \\
\text { Camarão- branco } \\
\text { Camarão- rosa } \\
\text { Camarão- } \\
\text { espinho/mãezinha } \\
\text { Búzios/ caramujo } \\
\text { Siri comum } \\
\text { Caranguejo goia, siri } \\
\text { relógio }\end{array}$ & $\begin{array}{l}\text { SUDESTE: } \\
\text { Anchieta, Piúma e Marataízes ES }\end{array}$ & OLIVEIRA, 2018. \\
\hline
\end{tabular}




\begin{tabular}{|c|c|c|c|c|}
\hline $\begin{array}{l}\text { Leucosiidae } \\
\text { Samaouelle, } \\
1819 \\
\text { Aethridae Dana } \\
\text {,1815 }\end{array}$ & $\begin{array}{l}\text { Petrochirus diogenes } \\
\text { (Linnaeus, 1758) } \\
\text { Callinectes ornatus } \\
\text { (Ordway, 1863) } \\
\text { Persephona punctata } \\
\text { (Linnaeus, 1758) } \\
\text { Hepatus pudibundus } \\
\text { (Herbst, 1785) }\end{array}$ & $\begin{array}{l}\text { Caranguejo goia, } \\
\text { dorminhoco }\end{array}$ & & \\
\hline $\begin{array}{l}\text { Penaeidae } \\
\text { Rafinesque, } 1815 \\
\text { Lysmatidae } \\
\text { Dana, } 1852 \\
\text { Diogenidae } \\
\text { Ortmann, } 1892 \\
\text { Portunidae } \\
\text { Rafinesque, } 1815 \\
\text { Leucosiidae } \\
\text { Samaouelle, } \\
\text { 1819 } \\
\text { Aethridae Dana } \\
\text {,1815 }\end{array}$ & $\begin{array}{l}\text { Xiphopenaeus kroyeri } \\
\text { (Heller, 1862) } \\
\text { Litopenaeus schimitti } \\
\text { (Burkenroad, 1936) } \\
\text { Farfantepenaeus } \\
\text { brasiliensis (Latreille, } \\
\text { 1817) } \\
\text { Exhippolysmata } \\
\text { oplophoroides } \\
\text { (Holthuis, 1948) } \\
\text { Petrochirus diogenes } \\
\text { (Linnaeus, 1758) } \\
\text { Callinectes ornatus } \\
\text { (Ordway, 1863) } \\
\text { Persephona punctata } \\
\text { (Linnaeus, 1758) } \\
\text { Hepatus pudibundus } \\
\text { (Herbst, 1785) }\end{array}$ & $\begin{array}{l}\text { Camarão sete- } \\
\text { barbas } \\
\text { Camarão- branco } \\
\text { Camarão- rosa } \\
\text { Camarão-espinho/ } \\
\text { mãezinha } \\
\text { Búzios/ caramujo } \\
\text { Siri comum } \\
\text { Caranguejo goia, siri } \\
\text { relógio } \\
\text { Caranguejo goia, } \\
\text { dorminhoco }\end{array}$ & $\begin{array}{l}\text { SUDESTE: } \\
\text { Anchieta, Piúma e Marataízes ES }\end{array}$ & $\begin{array}{l}\text { BRAGA et al., } \\
2018 .\end{array}$ \\
\hline $\begin{array}{l}\text { Penaeidae } \\
\text { Rafinesque, } 1815\end{array}$ & $\begin{array}{l}\text { Xiphopenaeus kroyeri } \\
\text { (Heller, 1862) }\end{array}$ & $\begin{array}{l}\text { Camarão sete- } \\
\text { barbas }\end{array}$ & $\begin{array}{l}\text { SUDESTE: } \\
\text { Anchieta e Barra Nova (ES) } \\
\text { NORDESTE: } \\
\text { Barras de Caravelas e Santa Cruz de } \\
\text { Cabrália (BA) }\end{array}$ & $\begin{array}{l}\text { MUSIELLO- } \\
\text { FERNANDES et al., } \\
2018 .\end{array}$ \\
\hline $\begin{array}{l}\text { Penaeidae } \\
\text { Rafinesque, } 1815\end{array}$ & $\begin{array}{l}\text { Xiphopenaeus kroyeri } \\
\text { (Heller, 1862) } \\
\text { Litopenaeus schimitti } \\
\text { (Burkenroad, 1936) }\end{array}$ & $\begin{array}{l}\text { Camarão sete- } \\
\text { barbas/ espigudo } \\
\text { Camarão-branco }\end{array}$ & $\begin{array}{l}\text { NORDESTE: } \\
\text { Lucena, Paraíba }\end{array}$ & $\begin{array}{l}\text { NASCIMENTO, } \\
2018 .\end{array}$ \\
\hline $\begin{array}{l}\text { Penaeidae } \\
\text { Rafinesque, } 1815\end{array}$ & $\begin{array}{l}\text { Xiphopenaeus kroyeri } \\
\text { (Heller, 1862) } \\
\text { Litopenaeus schimitti } \\
\text { (Burkenroad, 1936) }\end{array}$ & $\begin{array}{l}\text { Camarão sete- } \\
\text { barbas/ espigudo } \\
\text { Camarão-branco }\end{array}$ & $\begin{array}{l}\text { NORDESTE: } \\
\text { Lucena, Paraíba }\end{array}$ & $\begin{array}{l}\text { NASCIMENTO et } \\
\text { al., } 2018 .\end{array}$ \\
\hline $\begin{array}{l}\text { Penaeidae } \\
\text { Rafinesque, } 1815 \\
\text { Lysmatidae } \\
\text { Dana, } 1852 \\
\text { Palaemonidae } \\
\text { Rafinesque, } 1815\end{array}$ & $\begin{array}{l}\text { Xiphopenaeus kroyeri } \\
\text { (Heller, 1862) } \\
\text { Litopenaeus schimitti } \\
\text { (Burkenroad, 1936) } \\
\text { Exhippolysmata } \\
\text { oplophoroides } \\
\text { (Holthuis, 1948) } \\
\text { Nematopalaemon } \\
\text { schimitti (Holthuis, } \\
\text { 1950) }\end{array}$ & $\begin{array}{l}\text { Camarão sete- } \\
\text { barbas } \\
\text { Camarão-branco } \\
\text { Camarão espinho } \\
\begin{array}{l}\text { Camarão barriga } \\
\text { branca }\end{array}\end{array}$ & $\begin{array}{l}\text { NORDESTE: } \\
\text { Lucena, Paraíba }\end{array}$ & $\begin{array}{l}\text { NASCIMENTO et } \\
\text { al., } 2019 .\end{array}$ \\
\hline
\end{tabular}

\section{DISCUSSÃO}

Pesquisas etnobiológicas e etnoecológicas voltadas à pesca artesanal abrangem informações importantes sobre o conhecimento tradicional de pescadores e pescadoras construídos a partir das suas observações do ambiente, inclusive de espécies-alvo capturadas em pescarias (PERUCCHI, 2013). No Brasil, ainda são escassos os estudos voltados ao conhecimento tradicional de comunidades pesqueiras artesanais sobre camarões peneídeos e a carcinofauna acompanhante da pesca de arrasto em áreas costeiras e na plataforma continental. Por isso torna-se importante a realização de estudos com esta abordagem, já que a obtenção de dados sobre estes crustáceos junto aos atores locais pode incrementar informações já obtidas por pesquisadores incentivando ações de cogestão em que há participação dos envolvidos (CÔRTES et al., 
2014; ABREU et al., 2017).

Nesta revisão, ao comparar as regiões brasileiras onde os estudos etnobiológicos e etnoecológicos de camarões peneídoes e carcinofauna da plataforma continental foram realizados, a região Sudeste e Nordeste foram as mais representativas. Provavelmente por apresentar grupos de pesquisas especialistas sobre o assunto, bem como a possível ocorrência de incentivos de financiamentos para tais estudos. Pesquisas com esta temática tem despertado interesse na comunidade científica uma vez que carrega riqueza de detalhe inerente ao conhecimento da biodiversidade, utilização dos recursos naturais e a relação humana com o ambiente (CLAUZET et al., 2005; MARTINS et al., 2015; BRAGA et al., 2018). Além disso, essa abordagem científica traz informações importantes que podem ser utilizadas como ferramenta para o manejo pesqueiro e futuros planos de ação ou políticas públicas (BRAGA et al., 2018; NASCIMENTO et al., 2018).

Nos trabalhos revisados, os pescadores abordados afirmam a importância dos camarões peneídeos como espécie-alvo da pesca (MUSIELLO-FERNANDES et al., 2017; 2018; BRAGA et al., 2018; OLIVEIRA, 2018). Estes camarões são considerados pela literatura brasileira um recurso pesqueiro de grande importância econômica e social há pelo menos 20 anos (DI BENEDITTO, 2001; BRANCO, 2005; FREITAS NETTO et al., 2007; EUTRÓPIO et al., 2013; CALUMBY et al., 2016; SILVA-GONÇALVES et al., 2016). De acordo com os últimos informativos pesqueiros oficiais do Brasil, tal recurso compreende $67,5 \%$ do total de crustáceos capturados (BRASIL, 2011), além disso $80 \%$ da produção mundial corresponde aos camarões da família Penaeidae (BARBIERI et al., 2001).

Ainda, para os pescadores entrevistados, $X$. kroyeri é o recurso pesqueiro mais explorado, seguido de L. schmitti e $F$. brasiliensis. O camarão sete-barbas e o camarão rosa são os peneídeos mais capturados no país em que juntos compreendem cerca de $45 \%$ do total da produção de crustáceos marinhos no Brasil (D'INCAO et al., 2002; BRASIL, 2011). Além dessas espécies, o camarão branco é também bastante comercializado nas regiões Nordeste e Sul do Brasil (D'INCAO et al., 2002; NASCIMENTO et al., 2018; 2019). De acordo com Frank et al. (2019), apesar dos gêneros Farfantepenaeus e Litopenaeus ocorrerem em maior frequência na região Sul, a pesca é direcionada aos camarões rosa e sete-barbas.

Embora a pesca artesanal dirigida aos camarões da família Penaeidae seja uma atividade importante para subsistência familiar e abastecimento de muitos mercados de pescado ao longo do litoral brasileiro (FREITAS NETTO et al., 2007; EUTRÓPIO et al., 2013; CALUMBY et al., 2016; SILVA-GONÇALVES et al., 2016), pouco se conhece sobre a etnobiologia e etnoecologia das espécies exploradas. Esta ausência de informações pode prejudicar significativamente a avaliação do impacto da atividade sobre os estoques naturais (BEGOSSI, 2004; SILVANO, 2004). Desta forma, torna-se necessário a incorporação do conhecimento de pescadores e pescadoras para auxiliar em medidas de gerenciamento do recurso, garantindo assim seu uso sustentável (GOMES, 2015). Neste contexto, esta revisão evidencia a necessidade de estudos sobre conhecimento tradicional já que a participação de comunidades pesqueiras pode contribuir com o conhecimento sobre biologia e ecologia de espécies (CLAUZET et al., 2005; MARTINS et al., 2015).

Estudos relacionados à etnoecologia e etnobiologia de crustáceos reúnem informações relevantes 
sobre os hábitos de vida, habitat, dimorfismo sexual dos camarões peneídeos (ZEINEDDINE et al., 2015; BRAGA et al., 2018; NASCIMENTO, 2018; NASCIMENTO et al., 2018; OLIVEIRA, 2018; NASCIMENTO et al., 2019) e sua relação com fatores ambientais que interferem no período reprodutivo e recrutamento desses camarões (BRAGA et al., 2018; NASCIMENTO, 2018; NASCIMENTO et al., 2018; OLIVEIRA, 2018; NASCIMENTO et al., 2019). Braga et al. (2018) e Oliveira (2018) relataram que camarões sete-barbas, rosa e branco são considerados alvo na pesca artesanal do sul do estado do Espírito Santo, sudeste do Brasil, e que pescadores possuem conhecimento relevante sobre a biologia e ecologia tanto desses camarões quanto da carcinofauna acompanhante. Outros autores identificaram que o camarão sete-barbas (ALARCON et al., 2009; GOMES, 2015) e o camarão rosa (PERUCCHI, 2013) também são considerados por pescadores e pescadores como um dos recursos mais explorados nas três regiões (Nordeste, Sudeste e Sul). Além dos camarões peneídeos, outros crustáceos como os caranguejos, siris, ermitão (búzio/caramujo) e camarão carídeo (mãezinha/ camarão-espinho), também foram mencionados pelos pescadores, tal como registrados nos trabalhos de Braga et al. (2018) e Oliveira (2018).

Estudos pioneiros relacionados ao conhecimento tradicional de carcinofauna acompanhante descrevem informações relevantes quanto ao papel ecológico desses organismos. Caranguejos, siris, ermitão e camarão carídeo são identificados por atores locais que descrevem diferenças entre macho e fêmea, preferência de substrato, fase de desenvolvimento e áreas de ocorrência de filhotes (BRAGA et al., 2018; OLIVEIRA, 2018). Além disso, os entrevistados mencionam sobre a utilização dos siris (Callinectes sp.) como isca de peixes, denominando como "engodo" (BRAGA et al., 2018). O período do defeso também é abordado nos estudos em que pescadores e pescadoras consideram a suspensão desta pescaria necessária, mas afirmam que os períodos são inadequados para os estados do Espírito Santo (MUSIELLO-FERNANDES et al., 2017), Santa Catarina (BAIL et al., 2007) e região Nordeste (NASCIMENTO, 2018) do Brasil.

Todas as informações compiladas neste trabalho sobre o status de conhecimento etnobiológico e etnoecológico de camarões peneídeos e carcinofauna acompanhante da pesca de arrasto retratam a riqueza dos saberes tradicionais de atores locais. Além disso, o diálogo entre órgãos governamentais e regulamentadores da pesca, pesquisadores e atores locais pode estabelecer maior confiabilidade entre as partes e contribuir com a cogestão pesqueira (ABREU et al., 2017). Neste contexto, esta revisão propõe ações integrativas envolvendo as partes interessadas, tais como: a) Obter informações detalhadas sobre a biologia e ecologia das espécies alvo de camarões e da carcinofauna acompanhante ao longo da costa brasileira; b) Reunir informações dos pescadores e pescadoras sobre as variações abióticas e bióticas, em uma escala temporal, para auxiliar na avaliação dos impactos ambientais e das atividades sobre os estoques naturais; c) Determinar, juntamente com os conhecimentos tradicional e científico, as áreas de reprodução e recrutamento de juvenis de crustáceos; d) Elaborar uma rede colaborativa com reuniões frequentes respeitando a linguagem e disponibilidade de atores locais a fim de intensificar o diálogo entre gestores e comunidades pesqueiras e definir em conjunto regras de defeso; e) Incorporar os saberes tradicionais nas medidas de gerenciamento do recurso pesqueiro; f) Compreender como a carcinofauna acompanhante e os descartes de camarão podem ser reaproveitados na geração de renda das comunidades pesqueiras e; g) 
Incentivar atividades de sensibilização por meio de trocas de conhecimentos entre atores locais que atuam na pesca e pesquisadores com intuito de garantir o uso racional do recurso pesqueiro.

\section{CONCLUSÕES}

O presente estudo reuniu informações sobre a etnobiologia e etnoecologia de camarões peneídeos e carcinofauna acompanhante da pesca de arrasto em áreas costeiras e na plataforma continental do Brasil já descritas pela literatura. As informações obtidas via conhecimento tradicional de pescadores e pescadoras podem gerar informações relacionadas à biologia e ecologia destes animais. As regiões Sudeste e Nordeste contribuíram com a maior quantidade de estudos, provavelmente por apresentar grupos de pesquisas com maior experiência sobre a temática e oportunidade de financiamento de pesquisas.

Apesar dos estudos apresentarem diversas informações fornecidas via conhecimento tradicional ainda é necessária a realização de pesquisas que aprofundem mais em questões de etnobiologia e etnoecologia de Crustacea. De modo geral, o conjunto de conhecimento apontado pelos pescadores e pescadoras pode ser unido às informações obtidas por pesquisadores e que juntos podem atuar na cogestão pesqueira. Desta forma, as propostas apresentadas nesta revisão contribuem pela integração dos conhecimentos de comunidade pesqueiras, dos gestores responsáveis pelas políticas públicas e de pesquisadores a fim de incrementar ações de políticas públicas voltadas à sustentabilidade da prática pesqueira e manutenção da cultura local.

\section{REFERÊNCIAS}

ABREU, J. S.; DOMIT, C.; ZAPPES, C. A.. Is there dialogue between researchers and traditional community members? The importance of integration between traditional knowledge and scientific knowledge to coastal management. Ocean and Coastal Management, v.141, p.10-19, 2017. DOI: https://doi.org/10.1016/j.ocecoaman.2017.03.003

ABREU, J. S.; DI BENEDITTO, A. P. M.; MARTINS, A. S.; ZAPPES, C. A.. Artisanal fishing in the municipality of Guarapari, state of Espírito Santo, Brazil: An approach to the perception of fishermen working in small-scale fishing. Sociedade and Natureza, Uberlândia, v.32, p.56-71, 2020. DOI: https://doi.org/10.14393/SN-v32-2020-46923

ALARCON, D. T.; COSTA, R. C. S. D.; SCHIAVETTI, A.. Abordagem etnoecológica da pesca e captura de espécies não-alvo em Itacaré, Bahia (Brasil). Boletim do Instituto de Pesca, São Paulo, v.35, n.4, p.675-686, 2009.

BAIL, G. C.; BRANCO, J. O.. Pesca artesanal do camarão setebarbas: uma caracterização socioeconômica na Penha, SC. Brazilian Journal of Aquatic Science and Technology, v.11, n.2, p.25-32, 2007.

BARBIERI, R. C. J.; OSTRENKY, A. N.. Camarões Marinhos, reprodução, maturação e larvicultura. Viçosa: Aprenda Fácil, 2001.

BATISTA V. S.; ISAAC V. J.; VIANA, J. P.. Exploração e manejo dos recursos pesqueiros da Amazônia. In: RUFFINO, M. L.. A pesca e os recursos pesqueiros na Amazônia Brasileira.
Manaus: IBAMA, 2004.

BEGOSSI, A.. Introdução: ecologia humana. In: BEGOSSI, A.. Ecologia de Pescadores da Mata Atlântica e da Amazônia. São Paulo: HUCITEC, 2004. p.13-36.

BOTELHO, E. R. O.; SANTOS, M. C. F.; PONTES, A. C. P.. Algumas considerações sobre o uso da redinha na captura do caranguejo-uçá, Ucides cordatus (Linnaeus, 1763), no litoral sul de Pernambuco - Brasil. Boletim Técnico-Científico do CEPENE, v.8, n.1, p.55-71, 2000.

BRAGA, A. A.; ZAPPES, C. A.; OLIVEIRA, A. C. M.. Estudo do conhecimento tradicional de pescadores do litoral sul do Espírito Santo sobre a carcinofauna acompanhante da pesca de camarões. Brazilian Journal of Aquatic Science and Technology, Itajaí, v.22, n.2, p.1-11, 2018.

BRANCO, J. O.; FRACASSO, H. A. A.. Ocorrência e abundância da carcinofauna acompanhante na pesca do camarão setebarbas, Xiphopenaeus kroyeri Heller (Crustacea, Decapoda), na Armação do Itapocoroy, Penha, Santa Catarina, Brasil. Revista Brasileira de Zoologia, v.21, n.2, p.295-301, 2004.

BRANCO, J. O.; VERANI, J. R.. Pesca do camarão sete-barbas e sua fauna acompanhante, na Armação do Itapocoroy, Penha, SC. In: BRANCO, J. O.; MARENZI, A. W. C.. Bases ecológicas para um desenvolvimento sustentável: estudos de caso em Penha, SC. Itajaí: UNIVALI, 2006. p.153-170.

BRANCO, J. O.. Biologia e pesca do camarão sete barbas 
Xiphopenaeus kroyeri (Heller) (Crustacea, Penaeidae), na Armação do Itapocoroy, Penha, Santa Catarina, Brasil. Revista Brasileira de Zoologia, v.22, n.4, p.1050-1062, 2005.

BRASIL. Ministério da Pesca e Aquicultura. Boletim Estatístico da Pesca e Aquicultura. Brasília: MPA, 2011.

CALUMBY, J. A.; LIMA, R. S.; BONIFÁCIO, A. D.; SOARES, E. C.. Monitoramento da captura de camarões marinhos Litopenaeus schmitti, Farfantepenaeus subtilis e Xiphopenaeus kroyeri no município de Coruripe, estado de Alagoas, Brasil. Acta of Fisheries and Aquatic Resources, v.4, n.2, p.82-90, 2016. DOI:

https://doi:10.2312/ActaFish.2016.4.2.82-90

CLAUZET, M.; RAMIRES, M.; BARRELLA, W.. Pesca Artesanal e conhecimento local de duas populações caiçaras (Enseada do Mar Virado e Barra do Una) no Litoral de São Paulo, Brasil. Revista Multi Ciência, v.4, p.1-22, 2005.

CÔRTES, L. H. O.; ZAPPES, C. A.; DI BENEDITTO, A. P. M.. Ethnoecology, gathering techniques and traditional management of the crab Ucides cordatus Linnaeus, 1763 in a mangrove forest in south-eastern Brazil. Ocean and Coastal Management, v.93, p.129-138, 2014. DOI: https://doi.org/10.1016/j.ocecoaman.2014.03.021

CÔRTES, L. H. O.; ZAPPES, C. A.; DI BENEDITTO, A. P. M.. The crab harvest in a mangrove forest in south-eastern Brazil: insights about its maintenance in the long-term. Perspectives in Ecology and Conservation, v.16, p.113-118, 2018. DOI: https://doi.org/10.1016/j.pecon.2018.02.002

CÔRTES, L. H. O.; ZAPPES, C. A.; DI BENEDITTO, A. P. M. Sustainability of mangrove crab (Ucides cordatus) gathering in the southeast Brazil: A MESMIS-based assessment. Ocean and Coastal Management, v.179, p.1-10, 2019. DOI: https://doi.org/10.1016/i.ocecoaman.2019.104862

DI BENEDITTO, A. P.. A pesca artesanal na costa Norte do Rio de Janeiro. Bioikos, Campinas, v.15, n.2, p.103-107, 2001.

D'INCAO, F.; VALENTINI, H.; RODRIGUES L. F.. Avaliação da pesca de camarões nas regiões Sudeste e Sul do Brasil. 19651999. Atlântica, v.24, n.2, p.103-116, 2002.

DIAS NETO, J.. Proposta de plano Nacional de gestão para o uso sustentável de Camarões Marinhos do Brasil. Brasília: IBAMA, 2011.

DIEGUES, A. C.. A pesca construindo sociedades: leituras em antropologia marítima e pesqueira. Núcleo de apoio à pesquisa sobre populações tradicionais e áreas úmidas brasileiras. São Paulo: USP, 2004.

EUTRÓPIO, F. J.. Biologia do camarão Xiphopenaeus kroyeri (Dendobranchiata: Penaeidae) e a fauna acompanhante relacionada a sua pesca em Anchieta, Espírito Santo, Brasil. Dissertação (Mestrado em Ecologia de Ecossistema) Universidade de Vila Velha, Vila Velha, 2017.

EUTRÓPIO, F. J.; MARIANTE, F. L. F.; FERREIRA JUNIOR, P. D.; KROHLING, W.. Population parameters of the shrimp Xiphopenaeus kroyeri (Heller, 1862) (Crustacea, Penaeidae), caught by artisanal fisheries in Anchieta, Espírito Santo State. Acta Scientiarum, Biological Sciences, v.35, n.2, p.141-147, 2013. DOI: http://doi.10.4025/actascibiolsci.v35i2.13408

FRANK, K. M.; FILHO, J. V. D.. Pesca de camarões na região sul do Brasil. Revista Gestão e Sustentabilidade Ambiental, Florianópolis, v.8, n.3, p.499-520, 2019. DOI: https://doi.10.19177/rgsa.v8e32019499-520

FREITAS NETTO, R.; DI BENEDITTO, A. P. M.. Diversidade de artefatos da pesca artesanal marinha do Espírito Santo. Biotemas, Florianópolis, v.20, n.2, p.107-119, 2007.

FRIESINGER, S.; BERNATCHEZ, P.. Perceptions of Gulf of St. Lawrence coastal communities confronting environmental change: hazards and adaptation, Quebec, Canada. Ocean and Coastal Management, v.53, p.669-678, 2010. DOI: http://dx.doi.org/10.1016/j.ocecoaman.2010.09.001

GOMES, A. A.. Etnoecologia pesqueira e dinâmica da pesca artesanal do litoral centro-sul do estado de São Paulo: um enfoque sobre a influência das variáveis ambientais na produtividade pesqueira. Dissertação (Mestrado em Aquicultura e Pesca) - Instituto de Pesca, São Paulo, 2015.

GRAÇA-LOPES, R.; TOMÁS, A. R. G.; TUTUI, S. L. S.; SEVERINO-RODRIGUES, E.; PUZZI, A.. Fauna acompanhante da pesca camaroeira no litoral do Estado de São Paulo, Brasil. Boletim do Instituto de Pesca, v.28, n.2, p.173-188, 2002.

HANAZAKI, N.; CASTRO, F.; OLIVEIRA, V. G.; PERONI, N.. Between the sea and the land: the livelihood of estuarine people in southeastern Brazil. Ambiente e Sociedade, v.10, p.121-136, 2007.

HANAZAKI, N.. Conhecimento caiçara para o manejo de recursos naturais. In: ALBUQUERQUE, U. P.. Atualidades em etnobiologia e etnoecologia. Recife: Sociedade Brasileira de Etnobiologia e Etnoecologia, 2002. p.17-25.

IBAMA. Lagosta, Caranguejo-Uçá e Camarão-do-Nordeste. Coleção Meio Ambiente, Série Estudos-Pesca. Brasília: IBAMA, 1994.

JANKOWSKY, M.; PIRES, J. S. R.; NORDI, N.. Contribuição ao manejo participativo do caranguejo-uçá, Ucides cordatus (L., 1763), em Cananéia, SP. Boletim do Instituto de Pesca, São Paulo, v.32, n.2, p.221- 228, 2006.

MAGALHÃES, H. F.; COSTA NETO, E. M.; SCHIAVETTI, A.. Local knowledge of traditional fishermen on economically important crabs (Decapoda: Brachyura) in the city of Conde, Bahia State, Northeastern Brazil. Journal of Ethnobiology and Ethnomedicine, v.8, n.13, p.2-9, 2012.

MARTINS, N. G.; RODRIGUES, D. A.; RIBEIRO, G. M.; FREITAS, R. R.. Avaliação da atividade pesqueira numa comunidade de pescadores artesanais no Espírito Santo, Brasil. Revista de Gestão Costeira Integrada, v.15, n.2, p.265-275, 2015. DOI: http://doi:10.5894/rgci514

MOURÃO, J. S.; NORDI, N.. Pescadores, peixes, espaço e tempo: uma abordagem etnoecológica. Interciência, Caraca, v.31, n.4, p.1-7, 2006

MUSIELLO-FERNANDES, J.; ZAPPES, C. A.; HOSTIM-SILVA, M.. Small-scale shrimp fisheries on the Brazilian coast: Stakeholders perceptions of the closed season and 
integrated management. Ocean and Coastal Management, v.148, p.89-96, 2017. DOI:

http://dx.doi.org/10.1016/i.ocecoaman.2017.07.018

MUSIELLO-FERNANDES, J.; ZAPPES, C. A.; HOSTIM-SILVA, M. Small-scale fisheries of the Atlantic seabob shrimp (Xiphopenaeus kroyeri): continuity of commercialization and maintenance of the local culture through making public policies on the Brazilian Coast. Ocean and Coastal Management, v.155, p.76-82, 2018. DOI: https://doi.org/10.1016/j.ocecoaman.2018

NASCIMENTO, G. C. C.. Conhecimento ecológico local e dinâmica da pesca artesanal do camarão marinho (Penaeidae): subsídio para conservação. Tese (Doutorado em Desenvolvimento e Meio Ambiente) - Universidade Federal da Paraíba, João Pessoa, 2018.

NASCIMENTO, G. C. C.; CÓRDULA, E. B. L.; SILVEIRA, T. A.; SILVA, M. C. B. C.. Conhecimento etnoecológico na pesca artesanal do camarão marinho (Penaeidae): sinergia dos saberes. Revista Ethnoscientia, Botucatu, v.3, p.1-18, 2018. DOI: http://doi.org/10.22276/ethnoscientia.v3i0.191

NASCIMENTO, G. C. C.; CÓRDULA, E. B. L.; CRISPIM, M. C.. Pescadores de arrastão de praia do litoral norte da Paraíba: entre o êmico e o ético no ciclo reprodutivo dos camarões marinhos. Revista Etos, v.3, n.1, p.74-84, 2019. DOI: http://doi.org/10.33501/revetos.v3n1.2019.024

OLIVEIRA, A. C. M.. Aspectos etnobiológicos e etnoecológicos na pesca artesanal em três municípios do litoral sul do Espírito Santo. Dissertação (Mestrado em Oceanografia Ambiental) - Universidade Federal do Espírito Santo, Vitória, 2018.

OLIVEIRA, P. C.; DI BENEDITTO, A. P. M.; BULHÕES, E. M. R.; ZAPPES, C. A.. Artisanal Fishery versus Port Activity in Southern Brazil. Ocean and Coastal Management, v.129, p.49-57, 2016. DOI:

http://dx.doi.org/10.1016/j.ocecoaman.2016.05.005

PAZ, V. A.; BEGOSSI, A.. Ethnoichthyology of Gamboa fishermen of Sepetiba Bay, Brazil. Journal of Ethnobiology, v.16, n.2, p.157-168, 1996.

PERUCCHI, L. C.. Pescando conhecimento: o conhecimento ecológico local e a gestão dos ambientes pesqueiros no litoral norte do Rio Grande do Sul. Dissertação (Mestrado em Desenvolvimento Rural) - Universidade Federal do Rio Grande do Sul, Porto Alegre, 2013.

SEDREZ, M. C.; BRANCO, J. O.; FREITAS-JUNIOR, F.; MONTEIRO, H. S.; BARBIERI, E.. Ictiofauna acompanhante na pesca artesanal do camarão-sete-barbas (Xiphopenaeus kroyeri) no litoral Sul do Brasil. Biota Neotropica, v.13, n.1, p.165-173, 2013.

SILVA-GONÇALVES, R.; D'INCAO, F.. Perfil socioeconômico e laboral dos pescadores artesanais de camarão-rosa no complexo estuarino de Tramandaí (RS), Brasil. Boletim do Instituto da Pesca, v.42, n.2, p.387-401, 2016. DOI: http://doi.org/10.20950/1678-2305.2016v42n2p387

SILVANO, R. A. M.. Pesca Artesanal e Etnoictiologia. In: BEGOSSI, A.. Ecologia de Pescadores da Mata Atlântica e da Amazônia. São Paulo: HUCITEC, 2004. p.187-222.

SOUZA, K. M.; CASARINI, L. M.; HENRIQUES, M. B.; ARFELLI, C. A.; LOPES, R. G.. Viabilidade econômica da pesca de camarão sete-barbas com embarcação de pequeno porte na praia do Perequê, Guarujá, estado de São Paulo. Informações Econômicas, v.39, n.4, p.30-37, 2009.

ZAPPES, C. A.; OLIVEIRA, P. C.; DI BENEDITTO, A. P. M. Percepção de pescadores do norte fluminense sobre a viabilidade da pesca artesanal com a implantação de megaempreendimento portuário. Boletim do Instituto de Pesca, São Paulo, v.42, n.1, p.73-88, 2016. DOI: https://doi.org/10.5007/1678-2305.2016v42n1p73

ZEINEDDINE, G. C.; BARRELLA, W.; ROTUNDO, M.M.; CLAUZET, M.; RAMIRES, M.. Etnoecologia da pesca de camarões usados como isca viva na Barra do Una, Peruíbe (SP/Brasil). Revista Brasileira de Zoociências, v.16, p.67 - 83, 2015.

A CBPC - Companhia Brasileira de Produção Científica (CNPJ: 11.221.422/0001-03) detém os direitos materiais desta publicação. Os direitos referem-se à publicação do trabalho em qualquer parte do mundo, incluindo os direitos às renovaç̃ões, expansões e disseminações da contribuiç̃o, bem como outros direitos subsidiários. Todos os trabalhos publicados eletronicamente poderão posteriormente ser publicados em coletâneas impressas sob coordenação da Sustenere Publishing, da Companhia Brasileira de Produção Científica e seus parceiros autorizados. Os (as) autores (as) preservam os direitos autorais, mas não têm permissão para a publicação da contribuição em outro meio, impresso ou digital, em português ou em tradução. 